\title{
Neuromotricidad, Psicomotricidad y Motricidad. Nuevas aproximaciones metodológicas \\ Neuromotricity, Psychomotricity and Motor skills. New methodological approaches
}

Eliseo Andreu-Cabrera, Francisco Javier Romero-Naranjo

Universidad de Alicante (España)

Resumen: El propósito de este artículo es analizar la terminología relacionada con la motricidad humana, al objeto de proponer el neologismo de neuromotricidad como concepto del siglo XXI, que se diferencie conceptualmente de otros términos similares, como motricidad o psicomotricidad. Los avances en el estudio del cerebro y su relación con el movimiento, nos empuja a la creación de un área especial dentro de la Ciencia de la motricidad humana, con planteamientos nnovadores. El método BAPNE (Romero, 2004) se postula como una posibilidad para optimizar el rendimiento del cerebro en el ámbito de la motricidad humana. Así mismo, aportamos una pirámide explicativa que muestra gráficamente la jerarquía terminológica dentro del ámbito de la motricidad, tanto a nivel teórico como práctico.

Palabras clave: Neuromotricidad, psicomotricidad, motricidad, coordinación motora, aprendizaje motor.

Abstract: The purpose of this article is to analyse the terminology related to human motor skills in order to propose the neologism of neuromotor skills (Neuromotricity) as a concept for the 21st century, which is conceptually different from other similar terms such as motor skills or psychomotor skills. Advances in the study of the brain and its relationship with movement have led to the creation of a special area within the science of human motor skills, with innovative methodological approaches. The BAPNE method (Romero, 2004) is postulated as a possibility to optimise the performance of the brain in the field of human motor skills. We also provide an explanatory pyramid that graphically shows the terminological hierarchy within the field of motor skills, both on a theoretical and practical level.

Keywords: Neuromotor skills, Neuromotricity, psychomotricity, motor skills, motor coordination, motor learning.

\section{Introducción}

Desde nuestra perspectiva como profesionales de las Ciencias de la actividad física y el deporte, y basados en nuestra experiencia, creemos que se debe avanzar en el ámbito de la motricidad teórico-práctica. A partir de una extensa revisión bibliográfica, hemos detectado una problemática preocupante que se traduce en cierto estancamiento en cuanto la Ciencia de la motricidad humana. Del mismo modo, a pesar de los intentos por constituir una Ciencia, en el ámbito motor, no existe el consenso necesario para establecer un objeto de estudio único y aceptado por todos.

A partir de esta eterna discusión en nuestra área, han surgido numerosos autores y propuestas, lo que provoca cierta dispersión en cuanto a conceptos e ideas relacionados con el cuerpo y el movimiento. En ocasiones, términos como motricidad o psicomotricidad se emplean como sinónimos, dependiendo del autor que los utilice.

Fecha recepción: 16-06-21. Fecha de aceptación: 16-09-21

Eliseo Andreu Cabrera

eliseo.andreu@ua.es
De hecho, existe una gran pugna entre dos ramas de la Educación física, que tratan de justificar, cada uno desde su planteamiento, si la Ciencia de la actividad física y el deporte, es en sí Ciencia o pseudociencia, enriquecida esta última por las aportaciones de las hermanas mayores como la Psicología, la Biología, etc... El planteamiento humanista se enfrenta al planteamiento técnico del movimiento, los músculos y la cuantificación de los resultados.

Por otra parte, desde el siglo XIX, existen referencias a planteamientos educativos que hoy en día no se sostienen, puesto que el conocimiento ha avanzado, y aquello que en su momento era innovador, desde la óptica del siglo XXI, ha quedado desfasado.

En la actualidad, el conocimiento del cerebro y la tecnología ha sufrido un avance espectacular. Las teorías y planteamientos filosóficos de otras épocas se pueden refutar con evidencias científicas, con neuroimágenes, o con instrumentación precisa y evolucionada.

Por todo ello, a nuestro entender, asistimos con preocupación al estancamiento teórico y práctico dentro del ámbito de la motricidad humana. En los textos analizados, existe cierta tendencia a citar a los autores clá- 
sicos y emplear la terminología impuesta y proveniente de siglos pasados. Terminología que se pierde en debates estériles sobre la autoría original de ciertos vocablos, a pesar de que no se pueda demostrar o que resulten descontextualizados. Ejemplo de ello, sería el término psicomotor, supuestamente acuñado por Wallon, cuyo sentido original difiere mucho del que en la actualidad se le asigna.

Otro aspecto a resaltar es la resistencia del profesional del ámbito motor a abandonar los enfoques clásicos y adentrarse en la investigación, en las propuestas innovadoras relacionadas con la motricidad humana.

Como ejemplo, desde el punto de vista metodológico, podemos citar la psicomotricidad infantil trabajada mediante el cuento motor con aros y picas. ¿Por qué no ampliar la estimulación cerebral creando entornos tecnológicamente punteros más allá de los balones de gimnasia rítmica?. (Véase https:// www.pramafitness.com/). Nos preguntamos si el sistema ha ahogado la creatividad de nuestros profesionales o si rodearnos de autores clásicos nacidos en el siglo XIX proporciona la seguridad de que las cosas se están haciendo correctamente.

En el ámbito académico parece existir consenso acerca de que la acción motriz está presente en nuestra vida, tanto en el ámbito educativo o terapéutico, como en el deportivo o incluso en las actividades músico motoras. Como afirma Rigal (2006), el desarrollo motor se refiere a la evolución motriz de sus capacidades, en la que va aumentando la complejidad y el control sobre su motricidad a lo largo de la vida. La educación motriz, dentro de la Educación física, tiene como objetivo mejorar la coordinación motriz. De acuerdo con Rigal, el desarrollo psicomotor concierne a la adquisición de conocimiento mediante la acción motriz, que permite explorar el mundo y obtener información, crucial para la formación del pensamiento.

En línea con este enfoque, creemos que como profesionales de la Educación Física, debemos avanzar en el conocimiento del cerebro, órgano en el que se refleja previamente el movimiento que se desea realizar.

A principios del siglo XX, desde la neuropsiquiatría se demostró tras investigar ciertas patologías, la relación entre los trastornos motores y los trastornos mentales. Según los últimos estudios, la motricidad es una de las características del ser humano que más influye en los procesos de aprendizaje. Posee raíces filogenéticas en la propia historia de la evolución y desarrollo del ser humano, y fundamentos ontogénicos, en lo relativo a la formación y desarrollo de los individuos orgánicos, en su interacción con el medioambiente. Las recientes investigaciones parecen coincidir en que una estimulación temprana y rica, en las etapas sensibles de la evolución humana, facilitarán la maduración motriz y cognitiva. Por ello, la psicomotricidad es estudiada desde diferentes ámbitos, entre los que destacan la Neurofisiología, la Psicología, la Psiquiatría y la Psicobiología. En el ámbito académico existe consenso en cuanto a la concepción del ser humano como un ente global, cuyos ejes básicos son el cuerpo y el movimiento.

Vitor da Fonseca (1998), profesor catedrático de la Facultad de Motricidad Humana de Lisboa, es uno de los pioneros en la observación sistemática de la psicomotricidad. Según Da Fonseca (1998), el sistema psicomotor tiene sus base en la simetría del sistema nervioso (tronco cerebral, cerebelo, mesencéfalo y diencéfalo) y organiza la motricidad (tonicidad, equilibrio y lateralidad), en la mayoría de los vertebrados. En adición a lo que antecede, el autor sostiene que, únicamente en la especie humana, también influyen las estructuras asimétricas (hemisferios cerebrales), en el concepto corporal, su estructuración espacio-temporal y en las praxias global y fina.

Desde las Ciencias de la actividad física y el deporte, la polisemia existente en torno al término psicomotor, nos sugiere definir y acotar términos, aparentemente semejantes como son motricidad, psicomotricidad o neuromotricidad. Esta área especial de la neuroeducación física merece ser debatida, al menos, como nuevo enfoque educativo dentro de la Ciencia de la motricidad humana.

El presente artículo tiene como objetivo impulsar la discusión conceptual entre los profesionales de la motricidad, y fomentar la investigación en lo relativo al cerebro, sus funciones y su implicación en el acto motor.

\section{Origen y evolución del término psicomotricidad}

La psicomotricidad entiende al ser humano como una entidad global y como disciplina, nace para deshacer la dualidad planteada por el filósofo René Descartes (1596-1650): mente-cuerpo (cuerpo y alma). El concepto de psicomotricidad fue desarrollado inicialmente en Alemania, a mediados del siglo XIX, por Wilhelm Griesinger, fundador de la neuropsiquiatría. La referencia más antigua sobre el término psicomotor, la aporta Renard, K. (2019), citando a Jean-Michel Lehmans. Este autor, afirma que W. Griesinger (1843), utilizó el 
vocablo «psicomotor» como síntoma en la hipotonía de una persona deprimida.

Coincidimos con Berruezo (2006) en que los investigadores no se ponen de acuerdo sobre el origen de la psicomotricidad, asignando a diferentes autores y diferentes fechas, su autoría y nacimiento. Según Renard, el término psicomotor se volvió a utilizar alrededor de 1870 por parte de E. Hitzig, psiquiatra y G. Fritsch, anatomista, para nombrar regiones de la corteza cerebral relacionadas con áreas puramente motoras. Dichas zonas, estimuladas eléctricamente, causan contracciones musculares en la mitad opuesta del cuerpo. En 1880, Leonard Landois, siguiendo con la terminología psicomotriz, incide en que el área cortical envía la estimulación eléctrica a los músculos para ejecutar el acto motor, no obstante se desconoce el proceso interno entre la psique y las habilidades motoras. Descubre la importancia de la voluntad en el movimiento, y la relación entre el psiquismo y la motricidad. Trastornos como la histeria tendrían teóricamente, una causa orgánica (constitución anormal del sistema nervioso central). Posteriormente, la neuropsicología y la psicología comprobarán que los problemas psicomotores aparecen durante el desarrollo de la persona y su relación con el entorno.

En palabras de Da Fonseca, durante el siglo XIX se estudia el cuerpo desde el campo de la Neurología, tratando de comprender el funcionamiento del cerebro y sus estructuras neuronales. Del mismo modo, la Psiquiatría estudia la masa gris como origen de alguna patologías del ser humano. Desde el modelo psicoanalítico, Freud (1920) explica el desarrollo humano desde la importancia de lo corporal, el movimiento y la interacción social que proporciona la motricidad.

Ernest Dupré (1925), dentro del ámbito patológico, parece ser quien acuña el término psicomotricidad en sus estudios sobre la debilidad motora en población especial. Establece relaciones entre ciertas anomalías neurológicas y psíquicas, y algunos problemas en el desarrollo motor. En el ámbito científico y desde la psicología evolutiva, destaca Henri Wallon (1925) como precursor de la psicomotricidad, quien desarrolla los primeros métodos de reeducación psicomotriz.

Desde una orientación psicobiológica, Wallon defiende que el movimiento es la única expresión e instrumento de la psique. La vida psíquica está estructurada por actitudes y comportamientos, claramente influidos por la motricidad. Sus investigaciones influyeron en el pensamiento psicológico de la década de los 70. En 1935, Guilmain publicó una de las primeras obras sobre reeducación de la psicomotricidad, titulada Fonctions psychomotrices et troubles du comportement. En ella, se analizan las funciones del sistema nervioso en relación a la . Edouard Guilmain diseñó ejercicios físicos que ayudaran a resolver dificultadespsicológicos en niños con problemas de escolarización (tanto motores como de carácter). Guilmain se inspiró en la obra de Henri Wallon y en 1948 publicó su libro Motor and Psychomotor Tests.

Ajuriaguerra (1960), discípulo de Wallon, consolida los principios y bases de la psicomotricidad. Ambos defienden que la psicomotricidad facilita el desarrollo y es el producto de la interacción entre los factores biológicos y los aspectos culturales. Afirman que desde una estrategia educativa, terapéutica y rehabilitadora, un entorno rico en estímulos, facilitará la maduración del sistema nervioso.Posteriormente, en el campo educativo, Jean Le Boulch (1966), crea la psicocinética o educación por el movimiento, siguiendo la obra de Wallon y Ajuriaguerra.

En 1967, se crea la Sociedad francesa de Educación y Reeducación psicomotriz, destacando entre sus creadores, André Lapierre (La Rééducation Physique,1951), Pierre Vayer y Bernard Acouturier. Su metodología de gimnasia correctiva consistió en ejercicios para la musculatura: musculatura dorsal, abdominal, de las extremidades inferiores y el arco plantar. Más adelante, añadieron ejercicios de distensión tónica asociada con los ejercicios de respiración. Desde una perspectiva genética, Piaget (1969) afirma que la motricidad interviene en la evolución de la inteligencia, a diferentes niveles en el desarrollo de las funciones cognitivas. Para este autor, citado por Ruiz Pérez (1987), todos los mecanismos cognoscitivos reposan en la motricidad.

En la escuela soviética, destacan algunos autores en el área de la psiconeurología del movimiento, como son Ozeretsky, Vygotsky (1962), Elkonin (1971) o Luria (1979). Estos investigadores inciden en la importancia del entorno social como origen del movimiento (sociomotricidad). Para los autores soviéticos, la motricidad humana es fundamental para el desarrollo infantil, que junto al lenguaje y al pensamiento, favorecen la adaptación a la sociedad. Concretamente Luria analizó la actividad psicológica que está detrás de la acción, y la estructura interna de la actividad mental, reflejada finalmente en la motricidad y el lenguaje humano. Según este autor, la organización neurológica (cortical) de la motricidad y el lenguaje, cambian con el aprendizaje.

Sarnat y Netsky (1981), citados por Fonseca (1998), sostienen que observando la neuroanatomía de los dife- 
rentes vertebrados, existe una gran complejidad en cuanto a la motricidad, y esto es debido a la evolución de las especies y su interacción con el entorno.

La relación entre el lenguaje y la motricidad ha sido estudiada por los autores más relevantes. Según Da Fonseca (1998), Wernicke (1848-1904), divide el cerebro en dos partes, la anterior o motora y la posterior, sensorial. Interpreta la afasia de Broca (afemia) como una pérdida de la imagen motora de las palabras y defiende la idea de que el córtex-motor contenía conceptos de movimiento (muy relacionado con la psicomotricidad).

En palabras de Oña (1994), el movimiento ha sido estudiado por las ciencias biológicas, concretamente desde la neurofisiología. Destaca el autor, los trabajos de Jackson (1870) sobre la función del córtex cerebral sobre la respuesta muscular o los de Herrick (1924) sobre la función del cerebelo en el movimiento humano. De acuerdo con Da Fonseca, la motricidad humana debe ser estudiada teniendo en cuenta la tonicidad muscular, el ajuste postural, el sistema vestibular que proporciona el equilibrio al cuerpo en el espacio, las funciones de memoria y procesamiento de la información, así como las aferencias del entorno, con los que programa la secuencia motora.

En la actualidad, la psiconeurología dispone de nuevas técnicas y tecnología, avances en neuroquímica y neurofarmacología, que permiten un diagnóstico y tratamiento más preciso y efectivo. En el ámbito educativo, según Fonseca, los conocimientos en psiconeurología deben ser aplicados a la educación y reeducación de los niños con necesidades especiales. Para ello, propone la psicomotricidad como herramienta.

Según el Ministerio de Educación de España, la neurociencia es la ciencia que estudia el sistema nervioso o ciencia del cerebro. Se ocupa de desvelar cómo funciona este complicado sistema y cómo produce la variedad de modelos de conductas que manifiesta nuestro organismo, incluidos los relacionados con los procesos cognitivos.

La neurociencia estudia la estructura, función y desarrollo del sistema nervioso, que sienta las bases de la cognición y del comportamiento. Desde la neurociencia cognitiva se estudia la complejidad del funcionamiento de la mente, de los millones de neuronas, su plasticidad y la influencia del ambiente en dicha red neuronal. La neurociencia cognitiva se centra en el análisis de la actividad cerebral en relación con la psique (consciencia), al objeto de entender el proceso biológico que condiciona el aprendizaje y la conducta. Por todo ello, esta ciencia se convierte en el marco para la investigación del comportamiento motor y de la neuromotricidad, como disciplina y método para el aprendizaje motor.

\section{Psicomotricidad: disciplina y método}

Pastor Pradillo (2002) diferencia dos significados del término psicomotricidad. Por un lado, como ciencia que se interesa por el estudio de la relación establecida entre lo psíquico y lo motor. Por otro, como método o técnica para conseguir diferentes objetivos motores. Ya sea como ciencia del movimiento, como metodología o como disciplina educativa, reeducativa y terapéutica, todos los investigadores parecen coincidir en que la psicomotricidad desarrolla la competencia motriz, la capacidad cognitiva y la inteligencia sociofectiva.

Miraflores\&Goldaracena (2021) recogen diferentes definiciones del término psicomotricidad. Destaca la de Boscaini (2002) quien la define como disciplina científica que atiende a la globalidad del hombre, a nivel psicocorporal, estructural y funcional, a través de la cual se busca el bienestar de la persona. Del mismo modo, recogen la definición de Bernaldo (2012), que la concibe como una disciplina que comprende a la persona en su globalidad, con sus aspectos motores y psíquicos, y cuya finalidad principal es el desarrollo de las competencias motrices, cognitivas y socio-afectivas.

En el primer Congreso Europeo de Psicomotricistas (1996), recogido por Berruezo (2000), se aportó la siguiente definición:

«Basado en una visión global de la persona, el término "psicomotricidad» integra las interacciones cognitivas, emocionales, simbólicas y sensoriomotrices en la capacidad de ser y de expresarse en un contexto psicosocial. La psicomotricidad, así definida, desempeña un papel fundamental en el desarrollo armónico de la personalidad. Partiendo de esta concepción se desarrollan distintas formas de intervención psicomotriz que encuentran su aplicación, cualquiera que sea la edad, en los ámbitos preventivo, educativo, reeducativo y terapéutico. Estas prácticas psicomotrices han de conducir a la formación, a la titulación y al perfeccionamiento profesionales y constituir cada vez más el objeto de investigaciones científicas.»

La Escuela Internacional de la psicomotricidad (2021) sostiene que la Psicomotricidad es la historia de la personalidad a través del lenguaje no verbal y del movimiento, entendiendo al individuo de una manera global que abarca lo físico, psíquico, social y cognitivo. Cuerpo y psique interactúan, elaborando la información externa (visual y táctil), producto de la interacción acción con el medio, con el que nos relacionamos de forma espon- 
tánea, imitativa, simbólica y cognitiva. Las emociones van unidas a los esquemas cognitivos y la Psicomotricidad tiene como objetivo fundamental comprender el vínculo que se establece entre psique y corporalidad. Bernaldo (2012) afirma que en Alemania, la psicomotricidad (psicomotorik) no existe con esa denominación, sino que la disciplina que estudia el movimiento se llama motología. Esta ciencia del movimiento, vinculada a las Ciencias de la actividad física y el deporte, está imbricada con la pedagogía (motopedagogía), la psicología (mototerapia rehabilitadora) y la medicina.

Desde la psicología cognitiva se pasa de un interés por la ejecución motora, al interés por los procesos internos. Los estudios de Posner (1969), citados por Oña, sobre la atención y la memoria en el control del movimiento, o los de Adams (1971) sobre el feedback sensorial en el aprendizaje motor, son ejemplos de dicha corriente.

Según Bernaldo (2012), en la década de los setenta, y en el ámbito metodológico, surgen dos corrientes que en la actualidad persisten: psicomotricidad dirigida y psicomotricidad vivenciada. La psicomotricidad dirigida también se conoce como instrumental, funcional, pedagógica o cognitiva, y se centra en los aspectos motores y cognitivos. Se basa en la aplicación de un test estandarizado, para realizar el diagnóstico y posteriormente aplicar técnicas para corregir el problema detectado. Miraflores \& Goldaracena (2021), proponen que para la psicomotricidad dirigida se utilizan las metodologías del juego, actividades o ejercicios motores, el cuento, el circuito motor y la canción motora.

Por otra parte, la psicomotricidad vivenciada, también llamada relacional o afectiva, se centra en el aspecto emocional (André Lapierre y Aucouturier, como pioneros), y se caracteriza por la motricidad espontánea. Miraflores \& Goldaracena (2021), recogiendo el criterio de diversos autores, resaltan que los contenidos a desarrollar dentro de la psicomotricidad vivenciada, son: la imagen corporal; la relación con el espacio; la relación con el tiempo; la relación con los objetos; y la relación con los otros. Además del placer de comunicar (escuchar y responder), el placer de crear (juego simbólico) y el placer de pensar, todo ello, mediante el juego y la actividad espontánea que surge dentro de la sala de psicomotricidad.

En los años ochenta se intenta consensuar los principios de la psicomotricidad como disciplina. Desde su origen, la discusión científica sobre la psicomotricidad ha sido profusa y constante, bien sea por tratar de definirla (como disciplina o como método), por acordar su objeto de estudio o por fijar sus funciones. Una de las definiciones más aceptadas es que la psicomotricidad es una ciencia que entiende al ser humano como ser integral y cuyo objetivo es el desarrollo cognitivo mediante el movimiento consciente. En otros términos, es la ciencia que concibe al ser humano como un ser integral, desde su corporeidad y que analiza la relación existente entre el desarrollo cognitivo, el movimiento, las emociones y la interacción con el medio en el que se relaciona y se expresa.

Según Van Manen (2003), citado por Águila y López (2019), el cuerpo vivido o corporeidad se refiere al hecho fenomenológico de que siempre estamos de una forma corpórea en el mundo. Al relacionarnos con los otros, el instrumento de convivencia es nuestro cuerpo, que nos permite experimentar con el entorno.

Este planteamiento se debe a Edmund Husserl (1900), fundador de la fenomenología trascendental, teoría filosófica que trata de describir cómo el mundo se hace presente en la subjetividad y cómo es su estructura. Tiene sus raíces en Descartes y su visión dualista del ser humano (cuerpo y alma), pero fue su alumno Merleau-Ponty (1945), quien defendió a principios del siglo XX, que la percepción tiene una dimensión activa, en la medida en la que representa una apertura al mundo de la vida. Ser consciente del cuerpo (corporalidad) es percibirlo de manera subjetiva y en relación al entorno y a los otros. El cuerpo, según Merleau-Ponty, constituye la apertura perceptiva al mundo y la propia creación de esa realidad, considerando el esquema corporal como expresión del cuerpo en el mundo.

Águila y López (2019), afirman que desde la segunda mitad del siglo XX, se cuestiona el cartesianismo y aparecen nuevas concepciones en las que el cuerpo toma un papel importante en la sociedad desde la corporeidad y la motricidad humana.

En el siglo XXI, ya se habla del cuerpo vivido que se entrena como ente biológico pero que es instrumento de exploración ambiental e interacción social. En este nuevo concepto de corporeidad, entran en juego los sentidos, las emociones, la parte afectiva del ser humano y por supuesto, la cognición. Mújica (2020) sostiene que otro aspecto difícil de transformar, es la realidad educativa que sucede en ciertas disciplinas pedagógicas, donde el cuerpo humano se le ha otorgado un lugar secundario. Este autor defiende que no existe ningún acto pedagógico que excluya la corporalidad.

Para Bernate (2021) la corporeidad toma un papel relevante en los procesos de formación, entendida como el ente mediador y las respectivas relaciones que se dan 
entre el conocimiento, el mundo que lo rodea y su integración con la naturaleza. Hurtado (2008), citado por Bernate (2021), afirma que desde la perspectiva fenomenológica, la corporeidad es el concepto clave desde el cual se ha construido esa forma de ordenamiento del conocimiento denominada Motricidad Humana.

Jean Le Boulch (1966) apostó por una ciencia del movimiento humano. Partió de la hipótesis de que el movimiento tenía una importancia fundamental en el desarrollo de la persona, por consiguiente, esta ciencia del movimiento no era una ciencia teórica donde se reflexiona sobre el movimiento, sino una ciencia aplica$\mathrm{da}$, es decir, que debe tener aplicación a todo lo que concierne al movimiento y a la enseñanza de la persona. A esto, el autor, lo denomina 'Ciencia del movimiento aplicada al desarrollo de la persona' .

Pierre Parlebas (1971) teorizó con la creación de una ciencia cuyo objeto de estudio es la acción motriz, siendo el referente de la Praxiología motriz.

Fue Manuel Sergio (1986), quien en su tesis doctoral «Para uma Epistemologia da Motricidade Humana», propone la Ciencia de la motricidad humana. La define como ciencia de la comprensión y de la explicación de las conductas motoras, orientada al estudio y a las constantes tendencias de la motricidad humana en el orden del desarrollo global del individuo y de la sociedad, teniendo como fundamento simultaneo lo físico, lo biológico y lo antropo/sociológico. Para este autor, la Ciencia de la motricidad humana se encuentra muy alejada de los planteamientos mecanicistas y biologistas y reconoce como características de los seres humanos, que la corporeidad es lo que los sujetos somos, y la motricidad lo que expresamos.

Oña (1994) realizó una excelente clasificación de las Ciencias de la motricidad, en la que las organizaba en función de unas perspectivas básicas: Física, Química, Biología, Psicología y Sociología. La Física se ocupa de estudiar la fuerza en el movimiento y la relación entre los diferentes segmentos corporales. La Química es la encargada de analizar las reacciones intracelulares en la ejecución de los movimientos. Del mismo modo, la Biología es la ciencia que estudia los órganos o tejidos implicados en la acción motora. Desde la Psicología, se analizan los procesos básicos de comportamiento en la respuesta motriz. Finalmente, la Sociología estudia los aspectos sociales implicados en el movimiento humano.

Dentro de estas perspectivas generales surgen áreas especiales como la Cineantropometría (Cinemática), la Biomecánica (Mecánica muscular), la Bioquímica del ejercicio (Neurofisiología, Medicina deportiva), la Sociología de la motricidad (Antropología, Sociología del deporte) y el Comportamiento motor (Desarrollo y aprendizaje motor).

Gómez Rijo et al. (2021) analizan la conducta motriz como objeto de estudio de la Ciencia de la motricidad humana. En una visión amplia del concepto, afirman que la conducta motriz contiene las dimensiones biológica, afectiva, cognitiva, interactiva y ejecución práxica. Para estos autores la dimensión biológica la constituyen aspectos físicos y mecánicos, es decir, la biomecánica, la fisiología, la anatomía y la neurología. En cuanto a la dimensión afectiva incluyen las emociones, pasiones y sentimientos y sostienen que para el desarrollo de la conducta motriz es esencial generar la cognición y la emoción humana a partir del registro sensorial del mundo externo y de la significación corporal. La dimensión interactiva incluye la relación consigo mismo, con otras personas y con el entorno o medio físico.

Es resaltable la aportación de Mora (2017), citado por Gómez Rijo et al. (2021), quien propugna que la dimensión cognitiva se refiere a la inteligencia motriz, que permite la realización práctica de una acción de forma repetitiva, concomitante o creativa. El cerebro humano posee áreas donde se elaboran los programas motores con los que se ejecuta la conducta motriz. En relación a la neuropsicología cognitiva, citan a Da Fonseca (1998), quien afirma que el sistema práxico contiene un almacén de fórmulas del movimiento y un mecanismo encargado de transformarlas en patrones inervatorios motores, que activan los componentes correspondientes del aparato motor y que afectan tanto a los movimientos con objetos como a las acciones expresivas, simbólicas y comunicativas.

Según Vitor da Fonseca (1998), la psicomotricidad es la integración interdisciplinar de áreas como la antropología, filogenética, ontogenética, cibernética y psiconeurología. Las relaciones entre la psicomotricidad y el aprendizaje están interrelacionadas en términos de desarrollo psiconeurológico.

En palabras de Cobos (1995), citado por Miraflores \& Goldaracena (2021), hablar de psicomotricidad es hablar de la globalidad del ser humano, su unidad psicosomática, la íntima relación entre su estructura somática, afectiva y cognitiva.

La psicomotricidad entiende al ser humano como un ser multidimensional (dimensiones psicosomáticas) y tiene como objetivo el desarrollo intelectual mediante el uso del movimiento consciente y voluntario, como 
medio de conocimiento del propio cuerpo, de las personas y del entorno. Chokler (1988), en Pastor (2002) define psicomotricidad como a disciplina que estudia al hombre desde una articulación intersistémica (sistemas anatomofisiológicos, psicológicos y sociales) decodificando el campo de significaciones generadas por el cuerpo y el movimiento en relación y que constituyen las señales de su salud, de su desarrollo, de sus posibilidades de aprendizaje e inserción social activa; y también las señales de enfermedad, de la discapacidad y de la imaginación. De Lièvre y Staes (1992), afirman que la Psicomotricidad es una aproximación global de la persona que puede incluir dos consideraciones: una función del ser humano que sintetiza psiquismo y motricidad con el fin de posibilitar una más eficaz adaptación; y una técnica que utiliza como instrumento específico el cuerpo, el espacio y el tiempo y que organiza la conducta del ser humano de manera que le facilite el conocimiento de sí mismo, de su entorno y la forma más adaptada de su actuar.

Pastor (2002) nos ofrece diversas definiciones como la de Pertejo y Manning (1983), quienes definen psicomotricidad como el estudio de la evolución y forma de manifestarse las vivencias del individuo consigo mismo y con el medioambiente, expresadas a través del cuerpo. Un cuerpo que ha de considerarse como órganos de expresión, de relación y de comunicación.

Lagrange (1983) define psicomotricidad como la educación del niño en su globalidad, porque actúa conjuntamente sobre sus diferentes comportamientos: intelectuales, afectivos, sociales y motores. Muniaín (1997) afirma que es una disciplina educativa/reeducativa/terapéutica, concebida como diálogo, que considera al ser humano como una unidad psicosomática, y que actúa sobre la totalidad por medio del cuerpo y del movimiento activos de mediación principalmente corporal, con el fin de contribuir a su desarrollo integral.

Según Pastor Pradillo (2002) desde una perspectiva educativa, psicomotricidad se identifica con una metodología integral que se caracteriza por la utilización de la actividad corporal para favorecer el desarrollo armonioso de los diferentes aspectos de la personalidad. Este autor ofrece una excelente recopilación de definiciones como la de Morales y García (1994), quienes afirman que desde una orientación conductual, definen la psicomotricidad como una técnica o conjunto de técnicas que tienden a influir en el acto intencional o significativo, para estimularlo o modificarlo, utilizando como mediadores la actividad corporal y su expresión simbólica.
Para Ramos (1979) es una técnica que tiende a favorecer por el dominio corporal la relación y la comunicación que el niño va a establecer con el mundo que le rodea. Defontaine (1982) la describe como una técnica que por medio del cuerpo y del movimiento, se dirige al ser en su totalidad. Quirós y Schrager (1979) concibe psicomotricidad como la educación o reeducación del movimiento, o por medio del movimiento que procura una mayor utilización de las capacidades psíquicas.

De acuerdo con Berruezo (1995), la psicomotricidad es un enfoque de la intervención educativa o terapéutica cuyo objetivo es el desarrollo de las posibilidades motrices, expresivas y creativas a partir del cuerpo, lo que le lleva a centrar su actividad e interés en el movimiento y el acto, incluyendo todo lo que se deriva de ello: disfunciones, patologías, estimulación, aprendizaje, etc.

Según recogen Miraflores\&Goldaracena (2021), las metodologías de aplicación práctica psicomotriz son muy variadas y suelen depender de las características de los participantes, teniendo en cuenta su edad, el nivel de madurez, sus preferencias, etc.

Mérida et al. (2018) resaltan que en las aulas infantiles, conocedoras de la necesidad de alcanzar un desarrollo integral que potencie equilibradamente las dimensiones físicas, emocionales, sociales y cognitivas de los niños y niñas, se ponen en marcha estrategias de psicomotricidad para favorecer el desarrollo de habilidades físicas como la coordinación general, el equilibrio y la motricidad gruesa.

Para Romero-Naranjo (2004) la psicomotricidad se puede trabajar con actividades músico motoras, creando el término «psicomotricidad rítmica» para aquellas actividades relacionadas con la percusión corporal a través del método BAPNE.

A partir de las definiciones de psicomotricidad que se han recopilado, proponemos la siguiente:

\section{* La psicomotricidad como disciplina}

Ciencia que entiende al ser humano como ser integral y multidimensional (física, social, cognitiva, emocional) y persigue su desarrollo armónico, integrando la psique y la motricidad consciente y voluntaria, para el desarrollo cognitivo basado en el conocimiento del propio cuerpo, del entorno y del resto de iguales.

\section{* La psicomotricidad como método}

La psicomotricidad es una metodología que según sus objetivos, puede ser educativa, reeducativa o terapéutica, y que está basada en técnicas y actividades lúdicas 
en las que se utiliza el cuerpo y el movimiento como ejes de acción, con el fin de contribuir a su desarrollo integral como persona.

\section{Neuromotricidad: concepto}

Entendemos motricidad como la capacidad del sistema nervioso central de producir la contracción de un músculo. También se define como capacidad de un cuerpo para moverse o producir movimiento.

En el ámbito de las Ciencias de la actividad física y el deporte, motricidad se refiere a un estudio de los movimientos del ser humano, sus características y significado. La motricidad como disciplina, estudia los movimientos humanos y sus características cinéticas y cinemáticas.

En un mundo en el que las tecnologías de la información y la comunicación rompen de manera clara en nuestros hábitos, y posiblemente, fomentan el sedentarismo (además de una baja estimulación cognitiva), es importante pensar en nuevos paradigmas que justifiquen otras formas de movernos y ser más efectivos. La relación entre movimiento, actividad física y funciones ejecutivas es una de las líneas de investigación que más han desarrollado en los últimos años, en diferentes ámbitos.

De la revisión documental realizada, se desprende que en el ámbito motor, el ejercicio físico es uno de los mejores protectores contra la demencia senil, la enfermedad de Alzheimer y la depresión.

Nuestro cuerpo y por ende, nuestro cerebro han sido creado para movernos. El ejercicio aumenta la producción de neurotrofinas, proteína encargada de ayudarnos a producir más neuronas, ayudando a tener un cerebro sano y flexible.

Ortiz y Ramírez (2020), destacan que existen evidencias científicas sobre la relación entre la actividad física de alta intensidad y los estudios de neuroimagen en niños, niñas y adolescentes. Concretamente sugieren la posible modulación de circuitos del cerebro que se relacionan con el control cognitivo, lo que ha permitido proponer que altos niveles de aptitud aeróbica pueden estar relacionados con un adecuado rendimiento académico.

Uno de los padres de la neurociencia es Santiago Ramón y Cajal (1906), premio Nobel de Medicina, cuyo descubrimiento de las hendiduras sinápticas, el espacio que separa las neuronas, gracias a los mensajeros químicos permiten la comunicación entre ellas. Este gran hallazgo sentó las bases del conocimiento sobre el fun- cionamiento del sistema nervioso central y periférico.

El movimiento consciente y voluntario y el lenguaje, entre otras, son características que nos distinguen como especie. Gracias al cuerpo y al movimiento, como ejes de la acción educativa, se desarrolla nuestro cerebro. La interacción humana y la estimulación del entorno provocan en el individuo, nuevas conexiones nerviosas.

Posteriormente, Ajuriaguerra (1947) fue el gran impulsor de la Neurología, la Neuropsiquiatría, la Psiquiatría Infantil, la Psicogeriatría y la Neuropsicología del Desarrollo.

En este campo, profundizó sobre las bases morfológicas, neurobiológicas y funcionales de las funciones psíquicas, las primeras organizaciones neuropsicológicas, la organización de la personalidad y la evolución de los funcionamientos neuropsicológicos en el niño.

Entre sus obras, destacan los libros Le Cortex cerebral. Étude neuro-psyco-pathologique (1949) y Manual de Psiquiatría Infantil (1977), y estudios sobre la desorganización motriz en grupos de dementes seniles con Alzheimer o sobre el tono muscular y sobre los problemas de integración de la percepción del cuerpo. Estos estudios abrieron la puerta a una nueva especialidad, la Psiconeurología, que posteriormente se denominaría Neuropsicología. Una especialidad de la neurociencia cuyo objeto es el estudio de la compleja red neuronal que organiza y controla el movimiento humano, es la neuromotricidad.

Lapierre (1974:40) en el capítulo Psicomotricidad y Neuromotricidad, señala que toda educación motriz es esencialmente psicomotriz, ya que el movimiento es el lazo de unión entre el pensamiento y la acción. Así mismo, sostiene que la psicomotricidad como método educativo, posee las características de consciencia y voluntariedad.

En adición a lo que antecede, cabe resaltar que la comunidad científica le otorga la autoría del término neuromotricidad, aunque solamente aparece como título del capítulo. Lapierre no aporta una definición ni como disciplina ni como método. No obstante, y en relación al cerebro y al movimiento, afirma que entre psiquismo y la mecánica de un músculo, existe toda una estructura neurológica de transmisión y regulación, que el autor denomina sistema psiconeuromotor. A partir de estos principios, Lapierre (1974) explica en detalle las bases neurofisiológicas esenciales que presiden la elaboración y ejecución de los movimientos voluntarios, automáticos y reflejos.

Según Hernando y Useros (2007) el desarrollo mo- 
tor del ser humano se produce en el marco de dos conceptos: el neuromotor y el psicomotor. Los procesos neuromotores y psicomotores se producen con una gran producción motórica en los primeros meses, después de nacer. En la actualidad se sabe que el proceso motor en su fase eferente, va unido a factores como la memoria motora, la atención, la percepción, la capacidad procedimental en el desarrollo del movimiento y otros componentes práxicos.

Gracias a los reflejos del recién nacido, cuyo origen proviene de un sistema nervioso inmaduro, el bebé aprende en su interacción con el mundo circundante. De acuerdo con Hernando y Useros (2007), el movimiento se basa en la respuesta adecuada y ordenada de todos nuestros componentes neuromusculares ante ciertos estímulos (interoceptivos y/o exteroceptivos) con un objetivo. Con la maduración del sistema nervioso, los reflejos se inhiben, logrando un repertorio motor coordinado, organizado, eficaz, económico, específico y funcional.

Desde las primeras patadas en el vientre de la madre, hasta la edad dorada del ser humano, la motricidad se desarrolla gracias al tono muscular, al equilibrio y a la coordinación. Todo bajo la organización de los circuitos neuronales y de todo un sistema de conexiones nerviosas, que condicionan la motricidad voluntaria, precisa y controlada. Es objetivo de la Neuromotricidad, el estudio de dicho proceso.

Dado que es una disciplina relativamente nueva, nos planteamos estudiar la producción científica existente sobre la neuromotricidad como disciplina o como método.

Tras una amplia búsqueda en las bases de datos de la Biblioteca de la Universidad deAlicante (19 Mayo 2021)*, encontramos que el término neuromotricidad ofrece escasos registros. En la tabla siguiente se exponen los resultados de dicha búsqueda. EnWOS (Neuromotricity - 9 registros).

\begin{tabular}{lcccc}
\hline Bases de datos & Neuromotricidad & Neuromotricité & Neuromotricidade & Neuromotricity \\
\hline Analytical Abstracts & 0 & 0 & 0 & 0 \\
ASSIA & 0 & 0 & 0 & 0 \\
Biblioteca Virtual en Salud & 0 & 0 & 0 & 0 \\
(BVS) & & & & \\
Web of Science & 0 & 0 & 0 & 9 \\
CINAHL & 0 & 0 & 0 & 1 \\
Cochrane & 0 & 0 & 0 & 0 \\
Csic & 0 & 0 & 0 & 0 \\
Cuadernos de pedagogía & 4 & 0 & 0 & 0 \\
CuIDEN & 0 & 0 & 0 & 0 \\
Dialnet & 9 & 0 & 0 & 6 \\
ERIC & 0 & 0 & 0 & 0 \\
Erihplus & 0 & 0 & 0 & 1 \\
JSTOR & 0 & 0 & 0 & 0 \\
MEDES & 0 & 0 & 0 & 0 \\
Pascal-Francis & 0 & 6 & 0 & 0 \\
Proquest central & 8 & 4 & 2 & 1 \\
Psicodoc & 0 & 0 & 0 & 0 \\
Psycinfo & 0 & 0 & 0 & 0 \\
Pubmed & 0 & 0 & 0 & 0 \\
Ulrich's & 0 & 0 & 0 & 0 \\
\hline *Colaboración de Ana B. Miquel Abril, Técnico documentalista de la Biblioteca de la Facultad de \\
Educación de la Universidad de Alicante. & & \\
& &
\end{tabular}

\section{Neuromotricidad como método}

Ros, E., Valcarcel, S., Jaikel, D., Berlai, S., Giglio, R., Payro, A., Romero-Naranjo, F.J. (2018). Attention in conservatoire students using body percussion following the BAPNE method. VIII International conference on intercultural education and international conference on transcultural health: the value of education and health for a global, transcultural world. European Proceedings of Social and Behavioural Sciences, 60, 448-455.

Castello, B., Anton, M.T. , Flores, N., Vicedo, M., Romero-Naranjo, F. J. (2018). Evaluating executive functions in primary school children in alicante using body percussion. VIII International conference on intercultural education and international conference on transcultural health: the value of education and health for a global, transcultural world. European Proceedings of Social and Behavioural Sciences, 60, 559-566.

Torro, R., Aparici, F., Arnau, A.F., Ulate, R.M., Cabrera, D.A., Romero-Naranjo, F. J. (2018). Pilot study into the executive functions of children aged 8-9 BAPNE method. VIII International conference on intercultural education and international conference on transcultural health: the value of education and health for a global, transcultural world. European Proceedings of Social and Behavioural Sciences, 60, 760-769.

Gonzalez, O. S., Romeu, C.E., Romero-Naranjo, F.J. (2018). Pilot study of executive functions in elderly adults in care homes. VIII International conference on intercultural education and international conference on transcultural health: the value of education and health for a global, transcultural world. European Proceedings of Social and Behavioural Sciences, 60, 770-776.

Cozzutti, G., Guaran, F., Blessano, E., RomeroNaranjo, F.J. (2018). Effects on executive functions in the BAPNE Method; a study on 8-9 years old children in Friuli Venezia Giulia, Italy. 7th International Conference on Intercultural Education - Education, Health and ICT - From a Transcultural Perspective. European Proceedings of Social and Behavioural Sciences, 237, 900-907.

\section{Término "neuromotricidad» citado en investigación}

Lakshmithathachar, M.A. (2010). Contribution of Ancient Indians to 'writing' (With Special Emphasis on South Asian and Indian Writing Systems). Proceedings 2010 12th International Conference on Frontiers in Handwriting Recognition (ICFHR 2010). P. 491. Indian Stat. Inst., Kolkata, India

Plamondon, R. (2010). Neuromuscular Studies of Handwriting Generation and Representation. Proceedings 2010 12th International Conference on 
Frontiers in Handwriting Recognition (ICFHR 2010). P. 261. Indian Stat. Inst., Kolkata, India.

Plamondon, R., Djioua, M., O’Reilly, C. (2009). Recent Developments in the Study of Rapid Human Movements with the Kinematic Theory. Traitement du signal. Vol 26, pp. 377-394.

Gasc, JP. (2001). Comparative aspects of gait, scaling and mechanics in mammals. Comparative biochemistry and physiology a-molecular \& integrative physiology. 21 st International Congress of the European-Societyof-Comparative-Physiology-and-Biochemistry.Vol. 131, pp. 121-133. Liege, Belgium.

En Cuadernos de Pedagogía (Neuromotricidad - 4 registros)

Neuromotricidad como método

(2021) CEIP Ciudad del mar. ABP (aprendizaje basado en proyectos) y neuromotricidad.

(2019) Fco. Javier Romero Naranjo. Método BAPNE como propuesta basada en la neuromotricidad.

Neuromotricidad como formación profesional

(2018) Oferta formativa sobre neuromotricidad. https : / / www. unir.net / educacion / cursopsicomotricidad-neuromotricidad/

Neuromotricidad como disciplina (definición)

(2017) Monográfico sobre actividad física y neurociencia. Ministerio de Educación. España.

En Dialnet (Neuromotricidad - 9 registros, Neuromotricity -6 registros. Dado que algunos documentos están en dos idiomas, el total de registros es 10)

Término "neuromotricidad» citado en investigación

Serna Carrión, M. (2020). Relación entre el desarrollo neuromotor y el lenguaje oral en Educación infantil. Journal of neuroeducation, 1, (1), 100-107.

Neuromotricidad como método

Romero Naranjo, F.J. (2020) Percusión corporal y solfeo cognitivo. Recursos pedagógicos según el método BAPNE. Pensamiento actual. 20, (35), 105-121.

Romero Naranjo, F.J. (2019) BAPNE for children. Canons and Rhythms: Neuromotricity and executive functions. 2-99 years old.

Romero Naranjo, F.J. (2019) Cognitive solfege: Step by step activities and rhythms

Romero Naranjo, F.J. (2019) BAPNE for children: 3-6 years old

Romero Naranjo, F.J. (2019) Africa for children: Neuromotricity and Executive functions. 2-99 years old.

Romero Naranjo, F.J. (2019) BAPNE for children \& Gross motor skills. Neuromotricity and Executive functions. 2-99 years old.

Romero Naranjo, F.J. (2019) Cognitive solfege: Neuromotricity and Executive functions. 2-99 years old.

Romero Naranjo, F.J. (2019) Cognitive solfege: Beat and motor control. 2-99 years old.

Romero Naranjo, F.J. (2019) BAPNE for children \& Fine motor skills. Neuromotricity and Executive functions. 2-99 years old.

En Erihplus (Neuromotricity - 1 registro)

"Neuromotricidad» como descriptor en revista española

Sportis. Scientific Journal of School Sport, Physical Education and Psychomotricity. Revista internacional para la difusión de la educación física, el deporte escolar y la psicomotricidad. Universidad de la Coruña. España.

ISSN: 2386-8333. https:// revistas.udc.es / index.php/SPORTIS/index

En Pascal-Francis (Neuromotricité - 6 registros)

Término "neuromotricidad» citado en investigación

Falk, B.; Usselman, C; Dotan, R. et al. (2009) Childadult differences in muscle strength and activation pattern during isometric elbow flexion and extensión. Applied physiology, nutrition and metabolism, 34, (4), 609. 615.

Vaivre-Douret, L.;Favarelle, I; Cabrol, D. et al. (2003) Le repérage de la douleur dórigine posturale chez le nouveau-né à terme en période néonatale: étude pilote $=$ Screening for posture-related pain once babies start walking: Pilot study. Revue internationale de pédiatrie, 324, 9-15.

Vaivre-Douret, L. (2006) Un outil normé pou l'évaluation des fonctions neuro-psychomotrices de lénfant: la Batterie NP-MOT= A standard tool for assessment of child neuro-psychomotor functions: The NP-MOT Battery. ANAE. Approche neuropsychologie des chez lénfant, 88-89, 237-240.

Mucchielli, A. (2009) Les prérequis neuropsychomoteurs fondamentaux contribuant à l'amélioration des apprentissages scoaires de base, à travers la méthode Ouros (Le Bon Départ): Psychomotricité et troubles neuropsychologiques=Basic neuropsychomotor skill prerequisites helping improve School learning through the Ouros method (For a good start). ANAE. Approche neuropsychologie des chez lénfant, 104-105, 439-447.

Vaivre-Douret, L. (2009) Contribution au diagnostic par l'évaluation standarisée des fonctions neuropsychomotrices chez l' enfant $=$ Contribution to the diagnosis by the standardized evaluation of the neuro- 
psychomotor child's functions. Evolutions psychomotrices, 83, 10-17.

Perfetti, C. \& Puccini, P. (1976) La progettualita assente. Neuromotricita e psicomotricita nella rieducazione del soggetto affetto da paralisi cerebrale infantile $=$ Absence de projection. Neuromotricité et psychomotricité dans la readaptation du sujet souffrant d'une paralysie cerebrale infantile. Neuropsichiatr. Infant. Ital. No 184-185, pp 1035-1040.

En ProQuest central (Neuromotricidad -8 registros, Neuromotricité -4 registros, Neuromotricidade -2 registros, Neuromotricity -1 registros)

Término "neuromotricidad» citado en Congreso internacional (virtual)

Graduados argentinos presentes en conferencia virtual Cubamotricidad. Prensa Latina Agencia Informativa Latinoamericana S.A.; Havana. 05 Dec 2020. Document URL: https://www.proquest.com/wirefeeds/graduados-argentinos-presentes-en-conferencia/ docview / 2470914192/se-2?accountid=17192

Neuromotricidad como método

De la Mora, Daniela (2019) O que a arte ensina. CE Noticias Financieras. Portuguese ed.; CENFPT. ContentEngine LLC, a Florida limited liability company . Miami. 7 Jan 2019.

De la Mora, Daniela (2019) Lo que el arte enseña. CE Noticias Financieras , Spanish ed.; CENFPT. ContentEngine LLC, a Florida limited liability company . Miami. 7 Jan 2019.

De la Mora, Daniela (2019) Lo que el arte enseña. CE Noticias Financieras , Spanish ed.; El Norte. Suplemento Softnews; Editora El Sol, S.A. de C.V. Monterrey, Mexico. 27 Jan 2019: 24.

Rodríguez, I. (2016) Percusión corporal: ritmo que ayuda al desarrollo del cerebro. La Nación. Miscellaneous; Grupo de Diarios América. San José, Costa Rica. 22 Oct 2016.

Rodríguez, A. (2016) En armonía. Reforma. Suplemento Softnews. Editora El Sol, S.A. de C.V. Mexico City. 25 Apr 2016: 17.

Término «neuromotricidad» citado en investigación

Vadillo, R.C. (2014) Integración e inclusión educativa vs. integración e inclusión excluyente: un desafío educativo. Revista Mexicana de Investigación Educativa; Consejo Mexicana de Investigación Educativa, 19, (63), 1285 1290.

Término "neuromotricidad» citado en prensa

Ce rapprochement de la prise en charge était urgent.
AllAfrica.com , French ed. SyndiGate Media Inc. Washington 05 Nov 2020. United States.

Bounoung, Par Yvan (2020). Ce rapprochement de la prise en charge était urgent. Cameroon Tribune; Yaounde. 05 Nov 2020. Cameroon.

Les «stakhanovistes du savoir» en quête de sens. Le Monde; Le Monde Interactif. Paris 29 May 2018.

Término «neuromotricidad» citado en investigación

Jasmin, E. (2013) Évaluation écosystemíque des besoins des enfants au primaire ayant un trouble de l'adquisition de la coordination (ou une dyspraxie) pour favoriser leur participation sociale. Thése doctoral. Dissertations Publishing. Université de Sherbrooke. Canada.

Padilha de Lima, A. \& Bruno Cardoso, F. (2017) A eficácia da estimulação cortical no desenvolvimento perceptivo motor de adolescentes com síndrome de Down. Universidade Nove de Julho (UNINOVE). ConScientiae Saúde, 16, (3), 311-317.

Silva, J.R.V et al. (2009) Sinergia entre o lóbulo frontal e parietal durante provas de coordenação motora global. Fitness \& Performance Journal, 8, (5), 360-365.

Gradenigo, B. (2002) Assessing and rehabilitation of car driving ability: A holistic approach. Europa Medicophysica, 38, (1), 33.

\section{La pirámide de la motricidad}

En función de los registros obtenidos tras la búsqueda en bases de datos, podemos afirmar que existen escasas definiciones de la neuromotricidad, ya sea como disciplina o como método. En concreto, en lo referente a la neuromotricidad como metodología, los registros encontrados no superan la veintena. Siguiendo el planteamiento de Oña (1994), resaltamos el surgimiento de la Neuromotricidad, como área especial de reciente creación.

El conocimiento del cerebro en el siglo XXI incide de manera inequívoca en nuevos procedimientos que fomenten la estimulación del córtex, la percepción, la programación del movimiento, el control motor y la toma de decisiones en el ámbito de la motricidad.

Finalmente, cabe señalar que el término «neuromotricidad» es citado en investigaciones relacionadas con el desarrollo y el aprendizaje motor, así como en estudios sobre aspectos neuromotores y cognitivos, especialmente en discapacidades o trastornos del desarrollo.

Al realizar una revisión conceptual de la terminología relacionada con la motricidad, nos centraremos en 
la neuromotricidad, como disciplina situada en la cúspide del trabajo motor. En la base de dicha pirámide se situaría la motricidad, como movimiento en sí, sin consciencia ni voluntariedad.

Le seguiría en el segundo escalón, la psicomotricidad, con las propiedades ampliamente analizadas en apartados anteriores, y fundamentalmente asimilada como disciplina y método del movimiento consciente con diferentes funciones relativas al desarrollo intelectual mediante la acción motriz.

En un tercer nivel de concreción, se sitúa la neuromotricidad como disciplina que estudia la relación entre las neurociencias y la motricidad, sin olvidar la parte psicomotriz del movimiento.

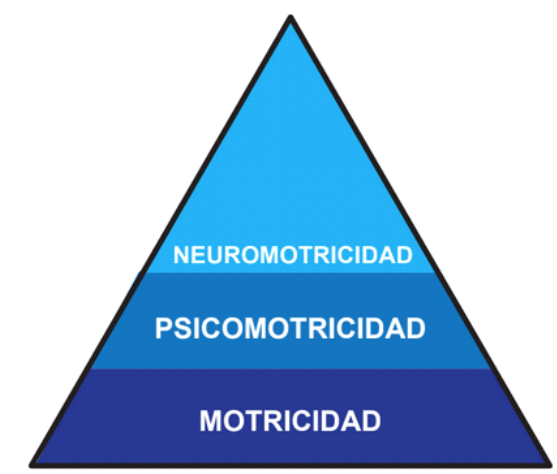

Figura 1. Pirámide de la neuromotricidad. (Romero-Naranjo y Andreu-Cabrera, 2021). Fuente: Elaboración propia.

Según Hernando y Useros (2007), la neuromotricidad es la respuesta nerviosa que desde los centros nerviosos corticales y subcorticales y que activa las motoneuronas para producir una respuesta motora.

La definición de neuromotricidad más reciente, la recoge Díaz Jara, experta en neuropsicología educativa, en Martín Lobo et al. (2015), monografía del MECD (Ministerio de Educación, Cultura y deporte) sobre Procesos e instrumentos de evaluación neuropsicológica educativa. Define neuromotricidad como análisis de los aspectos neurológicos que intervienen en el desarrollo de un movimiento, su programación, su control y la adquisición de los modelos de ejecución del mismo.

Desde el Ministerio de Educación y Formación profesional de España, definen neuromotricidad como la ciencia que estudia la relación entre las neurociencias y la motricidad, sin olvidar la parte psicomotriz del movimiento.

Tras la revisión de, proponemos dos definiciones.

\section{* Neuromotricidad como disciplina}

Parte de la Neurociencia que estudia los procesos neurológicos que influyen en la generación y dominio de la motricidad humana, y que planifican, organizan, evalúan y controlan el movimiento, para conseguir hitos motores en la adaptación al entorno y en función de la estimulación ambiental.

\section{* Neuromotricidad como método}

La neuromotricidad es la metodología educativa y neurorrehabilitadora dentro del ámbito motor, que incide en la estimulación cognitiva y socioemocional, mediante el trabajo específico de las funciones ejecutivas del cerebro, en relación al aprendizaje y la motricidad.

Una línea de trabajo podría ser el método BAPNE (Romero, 2004) que consiste en un plan de actividades diseñadas en progresión de dificultad psicomotora, para el trabajo de las funciones ejecutivas. A continuación, se detallan los contenidos de dicho plan de acción:

Velocidad de procesamiento: refleja la cantidad de información que puede ser procesada por unidad de tiempo o, incluso, la velocidad a la que puede realizarse una serie de operaciones cognitivas, pero también, el tiempo que transcurre desde la aparición del estímulo hasta la ejecución de una respuesta (Ríos et al., 2004). Puede ser visual (letras y números) auditiva (lenguaje y canto) y de movimiento. Se trabaja con las «Planchas Cognitivas BAPNE», el Clap Change, mediante estructuras pregunta/respuesta de texto orales rítmicas y melódicas, verbalizando operaciones matemáticas y moviéndonos en diferentes «metros horizontales» (figuras geométricas) en el espacio.

Memoria de trabajo. también denominada memoria operativa, es la capacidad de registrar, codificar, mantener y manipular la información durante un intervalo muy concreto de tiempo para mantener el sentido de unidad de la actividad cognitiva. En diversos tipos de actividades empleando repeticiones variadas y combinando nuevas estructuras rítmicas tanto verbales como con percusión corporal.

Fluidez verbal: relacionado con los procesos que llevan a cabo las estrategias adecuadas para la búsqueda de información y su respuesta apropiada en el menor tiempo posible. Se trabaja mediante la capacidad de improvisar verbalmente dentro de unas estructuras determinadas facilitadas por el docente. Se relaciona con dual task ya que se realiza a la vez que se está moviendo en unas figuras geométricas determinadas.

Dual task: es la capacidad de realizar dos tareas completamente diferentes simultáneamente y prestando igual atención a ambas de manera constante. Se trata de trabajar en paralelo una tarea visual y una visoespacial. En BAPNE todas las actividades presentan dual task ya 
que la finalidad es independizar extremidades inferiores de las superiores con movimientos diferentes y a la voz (hablada o cantada).

Inhibición o control de la interferencia: es la capacidad de inhibir o controlar las respuestas impulsivas, interferencias o distractores mientras se realiza una tarea. Se puede trabajar a nivel motor, atencional y conductual. Múltiples actividades relacionadas con melodías psicomotoras, canon, círculos concéntricos y reacción inversa.

Flexibilidad cognitiva: es la habilidad que permite realizar cambios en aquello que estaba previamente planeado y adaptarnos así a las circunstancias de nuestro entorno. El docente facilita constantemente indicaciones que modifican el movimiento psicomotor.

Planificación: es la capacidad de generar objetivos, desarrollar planes de acción para conseguirlos y elegir el más adecuado con base a la anticipación de consecuencias. Cuando se reciben las indicaciones de qué se ha de realizar a continuación, el participante desarrolla planes de acción para conseguirlo.

Toma de decisiones: es el proceso de realizar una elección entre varias posibles en función de las necesidades, valorando los resultados y consecuencias de cada una de ellas. Principalmente se trabaja con el Clap Change y Handsball Change.

Branching: es la capacidad de organizar y realizar tres tareas óptimamente de manera simultánea, intercalándolas y sabiendo en qué punto está cada una de ellas en cada momento. Cuando las extremidades inferiores se mueven realizando una figura geométrica, las extremidades superiores realizan Handsball Change y la voz entona, por ejemplo, en el 1er. tiempo Do, en el $3^{\circ} \mathrm{Mi}$ y en el $4^{\circ} \mathrm{Sol}$, siendo estas tareas cambiantes casi continuamente por el docente modificando la secuencia psicomotora.

\section{Conclusiones}

El objetivo de este artículo ha sido esclarecer desde el punto de vista terminológico, las principales acepciones vinculadas a la motricidad, psicomotricidad y neuromotricidad, con el propósito de ofrecer un recorrido histórico hasta la actualidad. En los últimos años, el conocimiento e investigación sobre el cerebro ha aportado nuevos canales de desarrollo, tomando especial relevancia la neuromotricidad.

En lo que se refiere al control motor, el vínculo con las funciones ejecutivas es fundamental, dado que es la «navaja suiza» que necesita nuestro cerebro para hacer frente a toda dificultad. Por este motivo, es fundamental conocer a nivel neuropsicológico los principios de la coordinación motora vinculados a las funciones cognitivas y las funciones ejecutivas. El conocimiento sobre el cerebro, por parte de los profesionales de la Educación física debe avanzar para no estancarse en metodologías didácticas o investigadoras, propias de otras épocas.

Coincidimos con Rodríguez y Hernández (2018), quienes defienden que la formación en el área de la motricidad, es parte de un contexto más amplio de necesaria innovación de modelos de formación (UNESCO, 2013). Estos autores destacan que desde la perspectiva de la Motricidad Humana, el desarrollo del ser humano se construye desde una unidad existencial indivisible, lo que implica contar con una formación didáctica específica en el currículum, para el diseño de experiencias que vinculen el hacer, sentir y pensar desde lo corpóreo, acorde con los principios pedagógicos propios de la educación infantil.

En este sentido, la neuromotricidad se integra en las Ciencias de la actividad física y el deporte. Hernández y De Barros (2021) defienden que las clases de Educación Física se convierten en un entorno natural adecuado para observar problemas neuronales asociados al área motriz. Sobre la preparación específica del profesorado, afirman que la formación en Neuroeducación Física permite a los docentes comprender mejor la situación de las personas con diversidad funcional por daños cerebrales.

Consideramos esencial que el educador posea una amplia base de conocimiento sobre el cerebro, ya que toda su acción educativa tiene un efecto directo sobre dicho órgano. El conocimiento neuropsicológico y neurocientífico facilitará el proceso de desarrollo de un alumno y mejorará las metodologías de intervención psicopedagógica.

A modo de conclusión, queremos propugnar que la Neuromotricidad, como disciplina y como método, se convierte en pleno siglo XXI, en núcleo común entre la Neurociencia y de las Ciencias de la actividad física y el deporte, con una visón transversal a otras disciplinas.

\section{Referencias}

Águila, C., y López, L.L. (2019). Cuerpo, corporeidad y educación: una mirada reflexiva desde la Educación Física. Retos, 35, 413-321.

Ajuriaguerra, J. (1960). Le Cortex Cérébral. Etude neurophycho-pathologique. Masson.

Álvarez del Villar, C. (1983) Preparación física del fútbol, a 
través del atletismo. Gymnos.

Benjumea, M.M. (2004). La motricidad, corporeidad y pedagogía del movimiento en educación física. Un asunto que invita a la transdisciplinariedad. En III Congreso Científico Latino Americano - I Simposio Latino Americano de Motricidad Humana. Universidad Metodista de Piracicaba UNIMEP. Brasil, abril de 2004.

Bernaldo, M. (2012). Psicología. Guía de evaluación e intervención. Ediciones Pirámide.

Bernate, J. (2021). Pedagogía y didáctica de la corporeidad. Una mirada desde la praxis. Retos, 42, 27-36.

Berruezo, P. P. (1995). El cuerpo, el desarrollo y la psicomotricidad. Psicomotricidad Revista de Estudios y Experiencias, 49, 15-26.

Berruezo, P.P. (2000). Hacia un marco conceptual de la psicomotricidad a partir del desarrollo de su práctica en Europa y en España. Revista Interuniversitaria de formación del profesorado, 37, 21-33.

Berruezo, P.P. (2000). El contenido de la psicomotricidad. En Bottini, P. (ed.) Psicomotricidad: prácticas y conceptos, 43-99.

Berruezo, P.P. (2006). Pasado, presente y futuro de la psicomotricidad. Monográfico de la Revista Iberoamericana de Psicomotricidad y Técnicas Corporales.VII Convención Iberoamericana de Estimulación y Psicomotricidad, 22, 25-36.

Cagigal, J.M. (1966). Deporte, pedagogía y humanismo. Comité Olímpico Español.

Da Fonseca, V. (1998). Manual de observación psicomotriz. INDE Publicaciones.

Calmels, D. (2011). ¿Qué es la psicomotricidad? Buenos Aires: Editorial Lumen.

De Lièvre y Staes (1992). Psychomotricité au service de l'enfant de l'adolescent et de l'adulte: Notions et applications pédagogiques. De Böck \& Belin. Broché.

Descartes, R. (1596-1650) Auteur du texte (1637). Discours de la méthode pour bien conduire sa raison et chercher la vérité dans les sciences, plus la dioptrique, les météores et la géométrie qui sont des essais de cette méthode. https: / /gallica.bnf.fr/ark:/12148/btv1b86069594/ f7.item

Dupré, E. (1925). Pathologie de l'imagination et de l'emotivité Payot.

Freud, S. (2001). Obras completas de Sigmund Freud.Volumen XVIII - Más allá del principio de placer, Psicología de la masas y análisis del yo, y otras obras (1920-1922). 1. Más allá del principio de placer (1920). Amorrortu editores.

Gómez, R.H. (2012). Del movimiento a la acción mo- triz: elementos para una genealogía de la motricidad. Educación Física y Ciencia, 14, 49-60.

Gómez Rijo, A. et al. (2021). (Re) pensar la competencia motriz. Retos, 40, 375-384.

González, A. \& González, C. (2010). Educación física desde la corporeidad y la motricidad. Hacia la Promoción de la Salud, 15 (2), 173-187

Guilmain, E. etWallon, H. (1935). Fonctions psychomotrices et troubles du comportement. Foyer central d'hygiène.

Hernández, A. \& De Barros, C. (2021). Inclusión, atención a la diversidad y neuroeducación en Educación Física. Retos, 41, 555-561.

Hernando, A. y Useros, A. (2007). Intervención fisioterápica en el proceso rehabilitador de pacientes con daño cerebral adquirido. Acción psicológica, 4 (3), 35-48

Hurtado, D. (2008) Corporeidad y motricidad. Una forma de mirar los saberes del cuerpo. Edicaçao \& Sociedade, 29(102), 119-136.

Landois, L. (1880). Lehrbuch der Physiologie des Menschen; (Digital 4th edition from 1885 by the University and State Library Düsseldorf.

Lapierre, A. (1974). La reeducación física (Tomo I, II, III). Científico-Médica.

Le Boulch, J. (1966). L’ Éducation par le mouvement : La psycho-cinétique à l'âge scolaire. Les editions sociales françaises.

Martín-Lobo, P. et al. (2015). Procesos y programas de neuropsicología educativa. Catálogo de publicaciones del Ministerio: mecd.gob. es Catálogo general de publicaciones oficiales: publicacionesoficiales.boe.es Ministerio de educación, cultura y deporte. Secretaría de Estado de Educación, Formación Profesional y Universidades. Centro Nacional de Innovación e Investigación Educativa (CNIIE).

Martín-Lobo, P. et al. (2015). Procesos e instrumentos de evaluación neuropsicológica educativa. Catálogo de publicaciones del Ministerio: mecd.gob.es Catálogo general de publicaciones oficiales: publicacionesoficiales.boe.es Ministerio de educación, cultura y deporte. Secretaría de Estado de Educación, Formación Profesional y Universidades. Centro Nacional de Innovación e Investigación Educativa (CNIIE).

Mérida, R. Et al. (2018). Descubrir el mundo con el cuerpo en la infancia. La importancia de los materiales en la psicomotricidad infantil. Retos, 34, 329 336.

Miraflores, E., y Goldaracena, I. (2021). Análisis de la psicomotricidad a través de la práctica psicomotriz 
de Bernard Aucouturier: estudio de casos. Retos, 39, 620-627.

Mujica Johnson, F.N. (2020). El término Educación Física en la postmodernidad: contribución de algunas perspectivas fenomenológicas. Retos, 38, 795-801.

Murcia, N; Corvetto, G. (2021). Motricidad y corporeidad como relaciones basadas en el desarrollo de lo humano. Cinta de Moebio, 70, 55-67.

Oña, A. (1994). Comportamiento motor. Bases psicológicas del movimiento humano. Servicio de Publicaciones de la Universidad de Granada.

Oña, A. (2007). Control y Aprendizaje Motor. Síntesis.

Ortiz, R., y Ramírez, M.L. (2020). Actividad, cognición y rendimiento escolar: una beve revisión desde las neurociencias. Retos, 38, 868-878.

Parlebas, P. (1971). Pour une épistemologie de l'Éducation Physique. Éducation Physique et Sport, 110, 1971.

Parlebas, P. (2001). Juegos, deporte y sociedad. Léxico de Praxiología Motriz. Paidotribo

Pastor, J. (2002). Fundamentación conceptual para la intervención psicomotriz en educación física. INDE.

Piaget, J (1969). El nacimiento de la inteligencia en el niño. Aguilar.

Renard, K. (2019). Repères historiques et chronologiques de la psychomotricité comme soin, site personnel internet de l'auteure, lescarnetsdesentiers.com, 19-02-2019, np.

Rey, A. \& Trigo, E (2000). Motricidad... ¿Quién eres?. Apunts. Educación Física y Deportes, 59, 91-98

Rigal, R.(1988). Motricidad humana. Fundamentos y aplicaciones pedagógicas. Pila Teleña.

Rigal, R. (2006). Educación Motriz y educación psicomotriz en preescolar y primaria. INDE.

Rodríguez, P.F. \& Hernández, F. (2018). Didáctica de la motricidad en la formación de profesores de educación infantil. Retos, 34, 25-32.

Romero-Naranjo, F.J. (2004). «Body Music-Body Percussion» Propuestas didácticas sobre psicomotricidad rítmica. Música y Educación. 17 (60), 53-80.

Romero-Naranjo, F.J. (2020). Percusión Corporal y «Solfeo Cognitivo». Recursos pedagógicos según el Método BAPNE. Pensamiento Actual, 20 (35), 105-121.

Ruiz Pérez, L. M. (1987). Desarrollo motor y actividades físicas. Gymnos.

Ruiz Pérez, L.M. (2007). Desarrollo, Comportamiento Motor y Deporte. Síntesis.

Ruiz Pérez, L.M. et al. (2008). El estudio del desarrollo motor: entre la tradición y el futuro. Fuentes, 8, 243-
258.

Schwartmann, A. (2006). La motricidad infantil: entre la experiencia corporal y el mundo exterior. Pensamiento Educativo, 38, 186-201.

Siedentop (1998). Aprender a enseñar la Educación Física. Inde.

Singer, R. (1986). El aprendizaje de las acciones motrices. Hispano europea.

Tefarikis, E. (2006). Motricidad Humana, un cambio urgente y necesario. Pensamiento Educativo, 38, 94-107.

Trigo, E. (2000). Fundamentos de la motricidad: aspectos teóricos, prácticos y didácticos. Gymnos Editorial Deportiva.

V.V.A.A. (2011). Intervenciones no farmacológicas. Reeducación psicomotriz. Instituto de Mayores y Servicios Sociales. Ministerio de Sanidad, Política Social e Igualdad. Secretaria General de Política Social y Consumo. Instituto de Mayores y Servicios Sociales (IMSERSO).

Viciana, J. \& Mayorga, D. (2018). The three-axes model of planning in physical education. Retos, 33, 313-319.

Wallon, H. (1925). L'enfant turbulent: étude sur les retards et les anomalies du développement moteur et mental. Quadrige. Presses universitaries de France. En: https: / /gallica.bnf.fr/ark:/12148/bpt6k3349523x/ f3.item.texteImage

\section{Enlaces}

http: / /www.educacionyfp.gob.es/dam/jcr:e173734d$070 \mathrm{~b}-4 \mathrm{f} 2 \mathrm{~b}-\mathrm{b} 8 \mathrm{c} \mathrm{c}-58 \mathrm{c}$ e 58 a d e $842 /$ bibliografia_neurmotricidad_aprendizaje_v1.pdf http: / / www.educacionyfp.gob.es/dam/jcr:4725269b06c4-4b61-bd29-bd9f3266d3bf/bibliografia-edinv1.pdf

http: / / www.educacionyfp.gob.es/dam/jcr:c912ff8eefdc-4a4d-bb8e-585991f2390c/bibliografiafejecutivas-v1.pdf

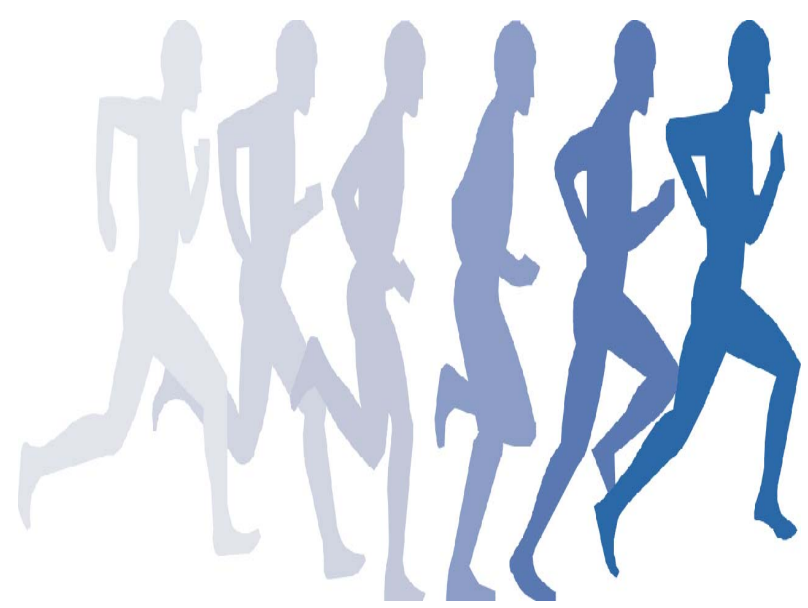

